http://dx.doi.org/10.18675/1981-8106.vol26.n52.p332-348

\title{
Programas de Intervenção Precoce: o que revelam as teses e dissertações
}

\author{
Early Intervention Programs: what to reveal dissertations and thesis
}

\section{Programas de la Intervención Temprana: lo que revelan las tesis y disertaciones}

\author{
Ana Regina Lucato Sigolo Candido' \\ Fabiana Cia" \\ 'Universidade Federal de São Carlos, São Paulo - Brasil. E-mail: ginasigolo@yahoo.com.br \\ "Universidade Federal de São Carlos, São Paulo - Brasil. E-mail: E-mail: fabianacia@hotmail.com
}

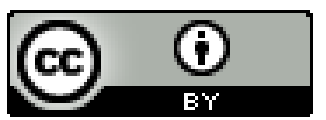

Educação: teoria e prática, Rio Claro, SP, Brasil - eISSN: 1981-8106

Está licenciada sob Licença Creative Common

\section{Resumo}

Os objetivos principais desse trabalho foram identificar as teses e dissertações produzidas em Programas de Pós-Graduação que abordam o tema da Intervenção Precoce e identificar nos estudos os dados empíricos que avaliam a relação entre os programas de intervenção precoce e as famílias. Os descritores utilizados foram atenção precoce, estimulação precoce, estimulação essencial, estimulação infantil, estimulação do desenvolvimento infantil, intervenção precoce e vigilância do desenvolvimento. Os trabalhos foram analisados por meio de uma ficha avaliativa em que constava o autor, instituição de origem, grau de titulação, tema principal, tipo de pesquisa, participantes, procedimentos para coleta de dados, análise dos dados, base teórica, fonte teórica, resultados e conclusões. Foram analisados 78 trabalhos. Os estudos revelaram que a identificação de crianças elegíveis para a estimulação precoce ainda está centrada nas características infantis. As avaliações têm se voltado para o desenvolvimento infantil sem considerar os fatores contextuais que também o influenciam.

Palavras-chave: Intervenção precoce. Estimulação precoce. Família. 


\begin{abstract}
The main objectives of this paper were to identify dissertations and thesis elaborated in Graduate Programs that approach the Early Intervention and to identify empirical data in the research that assess the relationship between early intervention programs and families. Early intervention, early stimulation, essential stimulation, infant stimulation, stimulation of child development, early intervention and monitoring of development were used as descriptors. The assessments had been analysed through an evaluation form in which counted the author, institution of origin, level of titles, main theme, type of research, participants, procedures for data collection, data analysis, theoretical basis, theoretical source, results and conclusions. Seventy-eight papers were analyzed and they have shown that identification of eligible children for early stimulation is still focused on infant characteristics. The assessments have focused on child development without considering the contextual factors that influence as well.
\end{abstract}

Keywords: Early intervention. Early stimulation. Family.

\title{
Resumen
}

Los principales objetivos de este estudio fueron identificar las tesis y disertaciones producidas en los Programas de Postgrado que abordan el tema de la intervención temprana e identificar los estudios de datos empíricos que evalúan la relación entre los programas y las familias de intervención temprana. Los descriptores utilizados fueron la intervención temprana, estimulación temprana, estimulación esencial, estimulación infantil, estimulación del desarrollo del niño, la intervención temprana y la vigilancia del desarrollo. Las obras fueron analizados por una hoja de evaluación que incluyó el autor, institución, nivel de títulos, tema principal, tipo de investigación, los participantes, los procedimientos para la recolección de datos, análisis de datos, base teórica, fuente teórica, los resultados y conclusiones. Se analizaron 78 estudios. Los estudios han demostrado que la identificación de niños elegibles para estimulación temprana todavía se centra en las características de los niños. Las críticas se han dirigido a desarrollo infantil sin considerar los factores contextuales que también influyen en ella.

Palabras clave: Revisión de la literatura. Intervención temprana. Familia.

\section{Introdução}

A intervenção precoce é efetiva em função do desenvolvimento apresentar muita plasticidade e o organismo humano ser considerado um sistema aberto influenciado pelo ambiente; e, na maioria das vezes, tem como objetivo remover sistematicamente os fatores externos de risco. Além disso, programas de intervenção precoce colocam crianças em ambientes enriquecidos para o seu desenvolvimento, treinam pais em responsividade e 
afetividade, oferecem um redirecionamento positivo contínuo e focam na construção de habilidades. Crianças que passam por programas de intervenção precoce têm maior probabilidade de no futuro completarem o ensino médio, de se manterem nos seus empregos, terem uma vida independente, evitarem a criminalidade e a gravidez na adolescência (GURALNICK, 1997).

A intervenção precoce tem por finalidade "capacitar a criança, por meio de apoio fornecido aos genitores e/ou outros cuidadores, a estabelecer uma trajetória de desenvolvimento que a preparará para ser bem sucedida, a longo prazo" (SILVA, DESSEN, 2005, p. 154).

Intervenção precoce pode ser concebida como uma abordagem multidisciplinar composta por um conjunto de recursos para crianças em risco ou com risco já adquirido que congrega a população de zero a seis anos e se caracteriza por diversas modalidades de intervenção, nomeadamente nos contextos familiares, educativos e, em outros locais de cuidado infantil. Aqui estão implícitos os três níveis de prevenção: primário, secundário e terciário (BAIRRÃO, 2003).

Os benefícios da intervenção precoce dependem claramente da detecção precoce, a qual requer que os profissionais, em especial os médicos, saibam como identificar acuradamente pacientes que têm deficiência ou atrasos no desenvolvimento. Em função das barreiras encontradas para a realização da identificação precoce de atrasos no desenvolvimento infantil, estes profissionais deveriam ter formas de identificar esses pacientes rapidamente (GLASCOE, 2000). De acordo com o estudo de Glascoe (2000a), nos Estados Unidos, 15 a $18 \%$ das crianças, aproximadamente, apresentam problemas de desenvolvimento e comportamento, e 7 a $10 \%$ apresentam uma forte experiência de fracasso escolar e, consequentemente, acabam desistindo da escola antes de terminarem o ensino médio. Desse modo, para garantir que as crianças tenham suas dificuldades identificadas precocemente e sejam encaminhadas para serviços especializados adequados, a American Academy os Pediatrics' Committee on Children with Disabilities recomenda que o pediatra utilize instrumentos de triagem validados para cada visita de supervisão de saúde. Nesta perspectiva, é possível se pensar na ampliação dessa prática para todos os profissionais da saúde.

A família teve por muito tempo um papel secundário no processo de educação de crianças pequenas em condição de risco no desenvolvimento. Bolsanello (2012, p. 13) afirma que até os anos de 1960 e 1970 "os programas de estimulação precoce eram destinados às crianças com deficiências na faixa etária entre zero e três anos e tinham como quadro de referência o modelo médico, centrado na relação profissional-criança, com a intervenção focada na deficiência infantil". As crianças eram vistas desvinculadas dos seus contextos de desenvolvimento, com definição de objetivos traçados pelos profissionais.

Portanto, os programas de intervenção precoce apresentavam como foco privilegiado exclusivamente a criança, desconsiderando o ambiente familiar e as influências de contextos 
mais amplos. Mesmo quando posteriormente a intervenção se voltou para a família da criança de risco, o modelo de atenção familiar era o patológico, ou seja, pressupunha que a condição deficitária estava presente, que todos os integrantes eram afetados negativamente e, finalmente que as adversidades familiares eram sempre explicadas por esta condição, desconsiderando o fato de que estas poderiam ser influenciadas por uma gama extensa de variáveis, o que poderia ocasionar uma diversidade de fontes de tensão: econômicas, de saúde, comunicativas, relacionais e que a dificuldade da criança seria apenas mais uma fonte de estresse.

A prevalência deste modelo de compreensão da família com uma criança de risco no desenvolvimento começa a ser reduzida por influência das pesquisas sobre famílias em geral, que substituem a visão estática por enfoques mais dinâmicos e interconectados entre os vários sistemas que a compõem e os contextos nos quais se desenvolve. Assim, na década de 1990, a emergência de concepções sistêmicas ${ }^{1}$ de desenvolvimento passa a influenciar os pressupostos dos programas de intervenção precoce, cujos objetivos de atuação contemplam as necessidades da criança e de sua família, considerando ainda o entorno no qual estão inseridas. O modelo de atuação se altera para o de colaboração entre família e profissionais (GINÉ et al., 2009).

De acordo com Bolsanello (2012, p.13):

Nas últimas décadas, sobretudo nos Estados Unidos e países europeus, os programas de estimulação precoce sofreram uma transformação notável, especialmente no que se refere à participação das famílias, que passou de um papel secundário a um papel de destaque nestes programas, e ao âmbito de atuação, que se estendeu para os serviços de saúde, sociais e educativos.

Esta mudança de perspectiva leva a uma atenção maior às necessidades em detrimento de suas carências e problemas, que requer, portanto, uma análise interativa, cujas dificuldades não são advindas apenas de determinados problemas da criança e outras condições familiares, mas também das características dos recursos que o ambiente lhes oferece ou deixa de fazê-lo.

Nesta perspectiva, as práticas empregadas nos programas de intervenção precoce evoluem para um modelo centrado na família, cujo interesse maior é atender às necessidades e expectativas do grupo familiar como um todo. Giné et al. (2009) defendem a perspectiva de intervenção que inclui o trabalho com famílias como um componente básico do marco de referência da atuação profissional.

Dessa forma, "a família como um todo deve ser considerada simultaneamente como receptora e agente ativo do programa" (ALMEIDA, 2004, p. 65). Esta perspectiva abrangente

\footnotetext{
${ }^{1}$ A perspectiva bioecológica de Bronfenbrenner e a transacional de Samerof.
} 
de intervenção tem por objetivo alcançar a melhoria da qualidade de vida da criança e da família.

Esta proposta tem como finalidade favorecer as condições para que a família alcance uma inserção adequada na comunidade, por meio do emprego adequado dos recursos disponíveis, aliada à construção de uma consistente rede social de apoio, considerando ainda a atenção à dificuldade/deficiência apresentada pela criança, principal justificativa para a proposição do programa. E como meta, a longo prazo, que a família consiga gerir os seus próprios recursos autonomamente de modo que o sentido de pertença à comunidade seja progressivamente consolidado.

As práticas centradas na família evidenciam a importância da tomada de decisão considerar as necessidades e recursos da criança, bem como dos pais, frente às prioridades da família. As oportunidades de aprendizagem para as crianças em contextos de vida diária, o apoio aos pais, a mobilização de recursos dos pais e da comunidade constituem em fatores promotores das habilidades da criança, dos pais e da família. Os apoios oferecidos podem abranger informações, aconselhamento, orientações que desenvolvem o conhecimento e a capacidade parentais para lidar com a própria criança e, com isso criar oportunidades de aprendizagem e desenvolvimento (CARPENTER, 2007; GINÉ et al., 2009).

Este movimento implica em uma mudança no modelo de atendimento passando de equipes multidisciplinares para interdisciplinares e de uma intervenção centrada exclusivamente na criança para uma proposta que considera a família e os contextos naturais de desenvolvimento (JUAN-VERA, PERÉZ-LOPÉZ, 2009).

Graça et al. (2010) afirmam que embora se reconheça a importância de intervenções centradas na família, a realidade ainda se mostra em um estágio aquém, cujos serviços ainda não funcionam em acordo com esta proposta e incorporá-la no trabalho cotidiano das equipes de profissionais nem sempre é uma tarefa fácil. Ainda, há toda uma retórica defendendo tal posição, mas o que se tem visto são programas voltados ao desenvolvimento de habilidades específicas com o envolvimento familiar limitado à participação materna.

A revisão de literatura pode se constituir em uma ferramenta que objetiva sintetizar os resultados consolidados sobre determinado tema, especificamente com o propósito de delimitar problemas de pesquisa que podem orientar novos rumos para a investigação científica na área da Intervenção Precoce. Em face deste amplo espectro de possibilidades, pareceu-nos adequado a opção de analisar as teses e dissertações produzidas nos programas de pós-graduação que versam sobre o desenvolvimento e aplicação de programas de intervenção precoce. 
Desse modo, os objetivos desse trabalho foram: 1) identificar as teses e dissertações produzidas em Programas de Pós-Graduação Brasileiros ${ }^{2}$ que abordam o tema da Intervenção Precoce; 2) analisar os principais resultados e as propostas decorrentes dos estudos identificados; 3) identificar os principais pressupostos teóricos que fundamentam os trabalhos; e 4) identificar nos estudos os dados empíricos que avaliam a relação entre os programas de intervenção precoce e as famílias.

\section{Método}

A modalidade de pesquisa bibliográfica denominada de revisão de literatura tem o propósito de resumir o conhecimento sobre um dado tema e permitir conclusões sobre a produção estudada (MANCINI, SAMPAIO, 2006). Dentre as características desse tipo de pesquisa ressalta-se a possibilidade de mapear e descrever um conjunto de trabalhos produzidos em locais legítimos de pesquisa os programas de pós-graduação; a definição de elementos metodológicos de acesso e caracterização da produção examinada e a disponibilidade de um conjunto de informações sistematizadas referentes a um grupo específico de trabalhos.

Nesta pesquisa foram identificados alguns descritores importantes para o objetivo proposto, a saber: atenção precoce, estimulação precoce, estimulação essencial, estimulação infantil, estimulação do desenvolvimento infantil, intervenção precoce e vigilância do desenvolvimento.

Foi realizada uma busca sistemática de cada descritor na condição expressão exata e, a partir disso, realizou-se a seleção de resumos que de fato estavam relacionados à investigação com crianças de até seis anos de idade em condição de risco (como condição de prematuridade e baixo peso), ou apresentassem uma deficiência (como auditiva, visual) ou transtornos invasivos do desenvolvimento (como autismo), ou ainda que objetivassem atuar em programas preventivos. Com base nos descritores identificados procedeu ao levantamento anual no período de 2003 a 2012.

Para análise dos resumos foi utilizada uma ficha avaliativa com a identificação das seguintes dimensões: autor, instituição de origem, grau de titulação, tema principal, objetivo, tipo de pesquisa, participantes, procedimentos para coleta de dados, análise dos dados, base teórica, fonte teórica, resultados e conclusões. Apoiado nos resultados do levantamento anual na condição "expressão exata" foram selecionados os trabalhos, cujos títulos dos trabalhos estavam relacionados com os objetivos dessa pesquisa.

\footnotetext{
${ }^{2} \mathrm{O}$ banco de dados de teses e dissertações da CAPES é constituído por trabalhos originais apresentados na íntegra tendo sido submetido a uma banca avaliadora. Dessa forma, artigos científicos e trabalhos apresentados em congressos podem representar um recorte desses trabalhos completos.
} 
A partir desse levantamento inicial foram retirados os títulos que apareceram em mais de um descritor. A etapa seguinte correspondeu à seleção dos trabalhos a partir de seus resumos. O critério para essa seleção foi definido a partir da leitura e análise desses trabalhos.

Ao final da análise dos resumos de acordo com a ficha avaliativa, foram discutidos os resultados aglutinados em algumas categorias, sendo elas: a) identificação precoce e elegibilidade que congrega as categorias de avaliação do desenvolvimento e/ou áreas específicas e identificação de fatores de risco/características infantis/habilidades; e b) as propostas de intervenção/estimulação.

\section{Resultados}

Os resultados apresentados estão divididos em 10 partes: (a) Grau de titulação e programas de pós-graduação; (b) Temas principais; (c) Tipos de pesquisa; (d) Participantes; (e) Procedimentos para coleta de dados; (f) Análise de dados; (g) Bases e Fontes teóricas; (h) Resultados; (i) Conclusões; e (j) Identificação precoce e elegibilidade; e Propostas de intervenção/estimulação.

\subsection{Grau de titulação e Programas de pós-graduação}

O total de trabalhos analisados foram 78; sendo 60 em nível de Mestrado, 15 em nível de Doutorado e três representaram os de Mestrado Profissional. Quanto à análise das áreas dos Programas de Pós-Graduação, mais da metade dos trabalhos se concentraram nas áreas da Educação (16), Psicologia (10), Educação Especial (7) e Saúde da Criança e do Adolescente (7). Os 38 trabalhos restantes se dividiram em 23 áreas diferentes de pesquisa.

\subsection{Temas principais}

Os temas mais frequentemente estudados nas pesquisas foram: proposta de intervenção/estimulação (30); concepções, expectativas e sentimentos de pais/profissionais sobre desenvolvimento/práticas (14); avaliação do desenvolvimento e/ou áreas específicas/atendimentos (10) e identificação de fatores de risco/características infantis/habilidades (10). Os outros 14 trabalhos investigaram temas como maternagem/parentalidade/cuidados maternos/paternos/familiares; interação social (criança/criança e criança/adulto); inclusão escolar de criança com deficiência; qualidade de vida e relações entre profissionais e mães/cuidadoras especiais. 


\subsection{Tipos de pesquisa}

A Tabela 1 mostra a frequência dos delineamentos de pesquisas por abordagem.

Tabela 1: Frequência do delineamento de pesquisa por abordagem

\section{Tipos de Pesquisa}

Quantitativa

Descritiva

Experimental

Correlacional/Levantamento

Intervenção/Quase-experimental

Correlacional

Comparativa

Experimental/Correlacional

Descritiva/Levantamento

Descritiva/Correlacional

Estudo de caso/Quase-experimental

Qualitativa

Descritiva

Estudo de caso

Intervenção

Exploratória

Etnográfica

Estudo de campo

Pesquisa-ação

Descritiva/Documental

\section{Número de Resumos}

31

9

8

3

3

2

2

1

1

1

1

34

13

8

5

4

1

1

1

1 
$\begin{array}{ll}\text { Mista } & 8\end{array}$

Descritiva 3

Intervenção 2

Pesquisa-ação 1

Comparativa 1

Levantamento 1

Bibliográfica 4

Não informada 1

Total 78

A Tabela 1 descreve os tipos de estudos encontrados. Grande parte das pesquisas se dividiu entre quantitativas (31 resumos) e qualitativas (34), seguidas por mista (8), bibliográfica (4) e em um estudo esta informação não foi descrita. Dentre os estudos quantitativos se destacaram os descritivos (9) e os experimentais (8). Já, os estudos qualitativos mais frequentes foram os descritivos (13), estudos de caso (8) e intervenção (5).

\section{Participantes}

A Tabela 2 mostra a frequência dos participantes das pesquisas.

Tabela 2: Frequência dos participantes das pesquisas

\begin{tabular}{ll}
\hline Relação de participantes & Número de Resumos
\end{tabular}

Bebês e crianças (trabalha com população típica/sem problema)

Crianças de risco/ com alguma deficiência (atrasos,

prematuridade, síndromes, deficiência)

Mães e crianças de risco/com deficiência

Mães /cuidadoras de crianças de risco/com deficiência 5

Famílias e crianças de risco/com deficiência

Profissionais da saúde e familiares (pais, mães, cuidadores) 5 
Profissionais da educação especial (professor de Educação

Especial/psico/to/fisio/fono)

Crianças de risco e não risco

$\begin{array}{ll}\text { Profissionais da educação especial e pais } & 2\end{array}$

$\begin{array}{ll}\text { Famílias e crianças } & 2\end{array}$

Residentes e graduandos (pediatria/psicologia) 2

Profissionais da saúde (agentes comunitários /enfermeiras) 1

Profissionais da educação (educadores/Pedagogo) 1

Profissionais da educação e crianças 1

Profissionais da educação e crianças de risco/com deficiência 1

Profissionais da educação, responsáveis, crianças e pares 1

Pais de crianças de risco/com deficiência $\quad 1$

Famílias

Profissionais da educação, mães e crianças 1

Mães, crianças de risco/com deficiência e babá 1

Famílias de crianças de risco/com deficiência $\quad 2$

Não informado

Não se aplica $\quad 5$

$\begin{array}{ll}\text { Total } & 78\end{array}$

Segundo dados da Tabela 2, os estudos selecionados apresentaram como participantes mais frequentes bebês e crianças (trabalha com população típica, sem problemas) (16), crianças de risco/com alguma deficiência (atrasos, prematuridade, síndromes, deficiências) (12), mães e crianças de risco/com deficiência (5), mães/cuidadoras de crianças de risco/com deficiência (5), famílias e crianças de risco/com deficiência (5) e profissionais da saúde e familiares (pais, mães, cuidadores). Em um trabalho a população estudada não foi descrita. 


\section{Procedimentos para coleta de dados}

Os procedimentos de coleta de dados mais empregados foram instrumentos de avaliação (10), entrevistas (8) e programas de estimulação/intervenção como medidas pré e pós-aplicação (7). Dado que chama atenção é que 12 resumos não informaram o procedimento de coleta de dados.

\section{Análise dos Dados}

A informação sobre a análise de dados foi pouco contemplada nos resumos, apenas 30 resumos a apresentaram. Destes 20 trabalhos empregaram a análise qualitativa identificada em alguns casos como análise de conteúdo, do discurso, processo reducional hermenêutico e método interpretativo psicanalítico, nove empregaram análises estatísticas e dois utilizaram métodos de análise quanti- qualitativos.

\section{Bases e Fontes teóricas}

As bases teóricas foram identificadas em apenas 20 resumos analisados, e as mais frequentes foram a psicanálise aplicada em 11 resumos e a psicologia do desenvolvimento em quatro. Apenas em 11 resumos foram explicitadas as fontes teóricas dos trabalhos.

\section{Resultados}

A Tabela 3 mostra os principais resultados encontrados nas pesquisas.

Tabela 3: Frequência dos principais resultados encontrados nas pesquisas

\begin{tabular}{cc}
\hline Categorias dos Resultados & Número de \\
Resumos
\end{tabular}

Efeitos positivos de programas de intervenção/capacitação de 20 profissionais

Correlação positiva entre fatores de risco (seja familiar, escolar, individual, de saúde) e problemas de desenvolvimento em geral e em áreas específicas

Sentimentos, significados e expectativas familiares em relação à criança de risco/com deficiência 
Desempenho da criança na avaliação do desenvolvimento

Não informado

Instrumentos de avaliação com propriedades psicométricas

adequadas (sensibilidade, especificidade)

Ausência de domínio de conhecimento específico sobre

Vigilância do Desenvolvimento Infantil/ sem direcionamento sobre práticas/práticas inadequadas sobre VDI

Predominância do modelo médico com o objetivo de atenuar os

efeitos da deficiência/participação da família secundarizada

Proposta de uma rede de suporte às crianças de risco/com deficiência

Não foram encontradas diferenças significativas na interação social entre crianças típicas e crianças de risco/com deficiência

Dificuldades dos profissionais de desenvolverem modelos de intervenção voltados para aspectos relacionais do conjunto pais-bebês

Não associação entre avaliação neurológica e resultados da escala cognitiva

Possibilidade de identificação de sinais de risco nos ambientes escolar e familiar

Programas de intervenção voltados para o envolvimento das famílias

Interação mãe-criança adequada

Condições de atendimentos das instituições

Serviços de avaliação/intervenção do desenvolvimento infantil descentralizados

Análise comparativa de programas de intervenção: programa

Currículo Funcional Natural foi mais efetivo que o TEACCH

Conduta dos profissionais quanto à detecção precoce 
A Tabela 3 elenca os principais resultados assinalados nos resumos dizem respeito aos efeitos positivos dos programas de intervenção/capacitação de profissionais (20), às correlações positivas entre fatores de risco (individuais, familiares e escolares) e problemas de desenvolvimento em áreas específicas ou em geral (12), aos sentimentos, significados e expectativas familiares/profissionais sobre a criança de risco/ com deficiência (8) e por fim, ao desempenho da criança na avaliação do desenvolvimento (7).

\section{Conclusões}

As principais conclusões assinaladas pelos trabalhos foram estratégias efetivas de intervenção (13), identificação de risco para o desenvolvimento (10), proposição de estratégias de prevenção/intervenção com a participação da família (9), associações entre resultados de avaliação de desenvolvimento e fatores de risco/ proteção (6) e análise das funções profissionais (6).

\subsection{Identificação precoce e elegibilidade; e Propostas de intervenção/estimulação}

Ao focalizar especificamente os estudos que versam sobre a identificação precoce e a elegibilidade para o serviço de intervenção precoce (total de 20 trabalhos), os instrumentos empregados para a avaliação do desenvolvimento neuropsicomotor foram: Escala Motora Infantil de Alberta (AIMS), Escala Bayley, Teste de Triagem de Denver, Escala de Desenvolvimento da Criança (EDC), Teste de Performance Motora do Lactente (TIMP), Inventário de Avaliação Pediátrica (PEDI), Teste de pré-triagem e Ficha do acompanhamento do desenvolvimento infantil.

Deste total, quatro analisaram as propriedades psicométricas de instrumentos quanto a sua sensibilidade de identificar atrasos ou problemas de desenvolvimento, seis trabalhos se propuseram a correlacionar os resultados da avaliação do desenvolvimento com fatores de risco ao nascimento, como baixo peso e prematuridade, com condições da própria criança como deficiência visual ou a presença de síndrome de Down e com fatores socioeconômicos familiares. Dois resumos objetivaram a avaliação neurológica voltada principalmente para condições de prematuridade em crianças pequenas. Os demais resumos tinham como propósito estudar alguma capacidade infantil como jogo simbólico em crianças com deficiência visual e noção espacial em crianças com síndrome de Down, identificação materna de indicadores de dor em crianças com paralisia cerebral e fatores de risco associados a estado nutricional, perda auditiva, surdocegueira e sofrimento psíquico.

A população alvo nestes estudos se referiu majoritariamente a crianças como: recémnascidos a termo e prematuros, no primeiro ano de vida, de um a quatro anos e em uma frequência bem menor crianças de cinco e seis anos de idade. Os pais aparecem como 
participantes em apenas dois estudos, em um como informantes das condições socioeconômicas em que vivem as crianças e em outro informante de uma ficha de acompanhamento do desenvolvimento infantil que tinha como objetivo de elegebilidade para programas de intervenção.

No tema "proposta de intervenção/estimulação" os trabalhos mais frequentes foram em relação ao desenvolvimento motor infantil e à intervenção precoce para autismo. Em relação ao autismo foram encontrados estudos sobre o desenvolvimento psicológico dessas crianças; ao itinerário terapêutico das famílias de crianças/jovens com TEA; uma pesquisa comparativa dos programas TEACCH e Currículo Funcional Natural quanto à interação social dessas crianças; e uma revisão bibliográfica sobre a eficácia EIBI (Early Intensive Behavioral Intervention).

Programas de capacitação em vigilância do desenvolvimento infantil (para enfermeiros, estudantes de psicologia e educadores de creches) também estavam presentes nesse tema. Além dessas propostas mais frequentes pode-se citar outras relacionadas ao desenvolvimento infantil; ao desenvolvimento afetivo, cognitivo e social; aos problemas de comportamentos (um estudo com destaque para os agressivos); intervenção domiciliar com pais (figura masculina) de crianças com síndrome de Down; ao trabalho fonoaudiológico com crianças deficientes visuais; ao fortalecimento do vínculo afetivo entre bebês e pais; em relação à consulta terapêutica como técnica de atendimento que pode interferir de forma positiva no desenvolvimento da criança; e, por fim uma revisão bibliográfica sobre a eficácia da estimulação precoce em crianças com SAF (Síndrome Alcoólica Fetal) principalmente em relação ao desenvolvimento motor e ao retardo mental.

Quanto à população alvo desses estudos pode-se dizer que a grande parte (cerca de 14 resumos) se concentrou no trabalho com crianças (os que citaram as idades abrangeram a faixa etária de zero a cinco anos e 10 meses) com características diversificadas como com desenvolvimento típico, deficiência visual congênita, de risco, autistas, com características contidas no TEA, com Síndrome Alcoólica Fetal e com síndrome de Down. A participação da família também foi encontrada com o foco nos responsáveis em dez trabalhos. Os profissionais englobados nessas pesquisas foram professores, fonoaudiólogos, babá, educadores de creches, fisioterapeutas, enfermeiros e graduandos em Psicologia, sendo que em quatro deles os participantes eram exclusivamente os profissionais.

Os resultados das dissertações e teses analisadas nesse tema mostraram que as propostas de programas de intervenção foram efetivas; que algumas variáveis podem interferir no desenvolvimento da criança como o desconhecimento da rede de reabilitação sobre o assunto e as discrepâncias do atendimento fisioterapêutico nos diferentes centros de atendimento; os ambientes escolar e familiar não proporcionam características estimuladoras apropriadas para crianças deficientes visuais comprometendo suas aptidões motoras e sociais.

Em relação aos trabalhos que focaram a população com características do TEA concluíram que as diretrizes clínico-educacionais são ferramentas importantes para nortear os 
profissionais em processos de intervenção; que o caminho terapêutico seguido pelas famílias normalmente não favorece a intervenção necessária para essa população, uma vez que a intervenção precoce é o procedimento mais eficaz para a melhoria desse quadro; que o Programa Currículo Funcional Natural se apresentou mais efetivo para a aquisição de comportamentos comunicativos de interação social e para a realização de atividades com instrução direta do professor pelas crianças quando comparado às que foram atendidas no Programa TEACCH; e que há evidências de eficácia do Programa EIBI.

Outras indicações decorrentes desses trabalhos foram a ênfase na necessidade de intervenção precoce no trabalho fonoaudiológico com crianças deficientes visuais, o trabalho em equipe e o acolhimento e/ou orientação aos profissionais que trabalham com essa população; o uso da estimulação precoce como ferramenta de inclusão social para a população em situação de risco em seu desenvolvimento; uma proposta de estratégias de intervenção para crianças de risco; e a substituição do modelo de intervenção centrado na criança pelo modelo centrado na família, sendo o pai uma figura importante para o funcionamento da família e desenvolvimento dos filhos.

\section{Discussão}

Os estudos revelaram que a identificação de crianças elegíveis para a estimulação precoce ainda está centrada nas características infantis quase que exclusivamente. As avaliações das crianças têm se voltado para o desenvolvimento infantil sem considerar os fatores contextuais que também o influenciam. No entanto, os programas de intervenção precoce parecem já se distanciar da perspectiva de que apenas as crianças devem ser alvo da intervenção, contudo, quando incluem outros agentes como familiares e profissionais ainda é no sentido de atender às demandas da criança, sem se preocupar com as da família. Embora se tenha identificado uma mudança, ainda se está distante do trabalho que se caracteriza como práticas de intervenção centradas na família (ALMEIDA, 2004; GRAÇA et al., 2010). Isto implica que estes ainda não refletem propriamente o avanço teórico da abordagem sistêmica que concebe a criança (em situação de risco que apresenta uma deficiência ou transtorno invasivo do desenvolvimento) inserida em uma rede de relações familiares que pode ser fonte de proteção ao seu desenvolvimento. Para tanto as pessoas que compõem o seu contexto imediato podem ser envolvidas no processo de intervenção para que o grupo familiar possa ser atendido em suas demandas (GINÉ et al., 2009).

A pulverização de áreas de conhecimento que investigam o tema da intervenção precoce indica a necessidade de propostas integrativas de diversas áreas, o que demanda a necessidade de se propor um modelo interdisciplinar de atendimento (JUAN-VERA; PÉREZLÓPEZ, 2009). Uma abordagem interdisciplinar se torna indispensável em função da influência no desenvolvimento infantil dos diversos contextos (familiar, educacional, de cuidados da saúde) que estão presentes na vida da criança (BAIRRÃO, 2003). 
Esta complexidade em tratar este tema leva a outra questão que poderia ser discutida como a baixa frequência de estudos que articulam uma proposta de intervenção, mesmo quando o descritor incide nesta mesma expressão. As inúmeras dificuldades impostas a este trabalho, muitas vezes, acabam por desanimar o pesquisador de se dedicar a esta tarefa. Trabalhos desta natureza acabam sendo realizados, mas não sob a forma de investigação o que pode, de alguma maneira, influenciar no avanço do conhecimento nas áreas de Intervenção Precoce. Destas hipóteses decorre a necessidade de sistematização de trabalhos na referida área.

Uma lacuna que também poderia ser citada é a escassez de estudos longitudinais que focam os benefícios que os programas de intervenção precoce podem proporcionar no âmbito econômico, social, da saúde pública, no desenvolvimento neuropsicomotor infantil, no desempenho escolar, entre outros. Uma vez que o caminho a ser percorrido é extenso e os resultados desses programas são a longo prazo (SILVA; DESSEN, 2005).

Para finalizar, do ponto de vista metodológico, o trabalho de revisão bibliográfica seria muito mais profícuo se as fontes de informação sobre a produção científica fossem totalmente confiáveis, com base em resumos que contemplassem as partes de uma pesquisa, com descrição precisa de objetivos, método de coleta e análise de dados, resultados e conclusões.

\section{Referências}

ALMEIDA, I. C. Intervenção precoce: focada na criança ou centrada na família e na comunidade? Revista Análise Psicológica, Lisboa, v. 22, n. 1, p. 65-72, 2004.

BAIRRÃO, J. Tendencias actuais em intervenção precoce. Psicologia, Porto, v. XVII, n. 1, p. 15-29, 2003.

BOLSANELlO, M. A. Apresentação. Educar em Revista, v. 43, p. 13-16, 2012.

CARPENTER, B. The ímpetus for family-centred early childhood intervention. Child: care, health and development, v. 6, p. 664-669, 2007.

DESSEN, M. A.; SILVA, N. L. P. A família e os programas de intervenção: tendências atuais. In: MENDES, E. G.; ALMEIDA, M. A.; WILLIAMS, L. C. A. (Orgs.). Temas em Educação Especial: avanços recentes. São Carlos: EDUFSCar, p. 179-187, 2004.

GINÉ, C.; GRÀCIA, M.; VILASECA, R.; BALCELLS, A. Trabajar con las familias en atención temprana. Revista Interuniversitaria de Formación del Profesorado, 65(23,2), p. 95-113, 2009. 
GLASCOE, F. P. Early Detection os Developmental and Behavioral Problems. Pediatrics in Review, v. 21, n. 8, p. 272-280, 2000.

GLASCOE, F. P. Detection and Addressing Developmental and Behavioral Problems in Primary Care. Pediatric Nursing, v. 26, n. 3, p. 251-266, 2000a.

GRAÇA, P. R. M.; TEIXEIRA, M. L. S. C.; LOPES, S. C. G; SERRANO, A. M. S. P. H.; CAMPOS, A. R. S. O momento da avaliação na intervenção precoce: o envolvimento da família. Estudo das qualidades psicométricas do ASQ-2 dos 30 aos 60 meses. Rev. Bras. Ed. Esp., Marília, v. 16, n. 2, p. 177-196, 2010.

GURALNICK, M. J. The Effectiveness of Early Intervention. Baltimore, MD: PF Brooks Publishing, 1997.

JUAN- VERA, M. J.; PÉREZ- LOPEZ, J. El funcionamento de um centro de desarollo infantil y atención temprana. Revista Interuniversitaria de Formación del Professorado, 65(23,2), p. 21-38, 2009.

MANCINI, M. C.; SAMPAIO, R. F. Editorial: Quando o objeto de estudo é a literatura: estudos de revisão. Revista Brasileira de Fisioterapia, São Carlos, v. 10, n. 4, p. 361, 2006.

SILVA, N. L. P.; DESSEN, M. A. Intervenção precoce e família: contribuições do modelo bioecológico de Bronfenbrenner. In: DESSEN, M. A.; JUNIOR, A. L. C. (Orgs.). A ciência do desenvolvimento humano: tendências atuais e perspectivas futuras. Porto Alegre: Artmed, p. 152-167, 2005.

Recebido em: 01/12/2014

Revisado em: 21/05/2015

Aprovado para publicação em: 27/05/2015

Publicado em: 31/08/2016 